\title{
Role of stem cell transplant and maintenance therapy in plasma cell disorders
}

$\sum_{\frac{1}{2}}^{2 C I E T Y O F}$

\author{
Philip L. McCarthy ${ }^{1}$ and Sarah A. Holstein ${ }^{2}$ \\ ${ }^{1}$ Department of Medicine, Blood and Marrow Transplant Program, Roswell Park Cancer Institute, Buffalo, NY; \\ and ${ }^{2}$ Department of Internal Medicine, Division of Oncology and Hematology, University of Nebraska Medical \\ Center, Omaha, NE
}

\begin{abstract}
Autologous stem cell transplant (ASCT) has been an important component of therapy for myeloma patients eligible for high-dose chemotherapy. Recent studies comparing early transplant to low-dose chemotherapy support the continued use of ASCT as consolidation following induction therapy, even in the era of immunomodulatory drugs, proteasome inhibitors, and other novel agents. Despite the marked improvements in outcomes with this approach, most patients will eventually experience disease progression. Thus, inclusion of post-ASCT consolidation/maintenance strategies is used to improve long-term disease control. Multiple randomized studies support the use of lenalidomide maintenance therapy following ASCT. The next generation of clinical trials will incorporate novel agents such as monoclonal antibodies, proteasome inhibitors, and other novel pathway modulatory agents into post-ASCT treatment strategies with the goal of achieving even deeper responses and longer durations of disease control.
\end{abstract}

\section{Learning Objectives}

- To understand the continued role of ASCT as consolidation following induction therapy in the era of novel agents

- To understand the role of post-ASCT maintenance therapy

\section{Introduction}

The plasma cell dyscrasias include a spectrum of disorders ranging from monoclonal gammopathy of undetermined significance, smoldering multiple myeloma, multiple myeloma, primary light-chain amyloidosis to solitary plasmacytoma. Other disorders associated with monoclonal gammopathies such as Waldenstrom macroglobulinemia and rare lymphomas will not be included in this section. Historically, treatment of plasma cell dyscrasias has been based on the presence of the CRAB criteria (hypercalcemia, renal dysfunction, anemia, and bone disease) and, more recently, these have been updated to include other criteria such as the serum free light-chain ratio and evidence of early bone disease based on more modern imaging techniques. ${ }^{1}$ Autologous stem cell transplant (ASCT) has been a mainstay of therapy for myeloma patients eligible for high-dose chemotherapy for decades. However, because nearly all patients will have disease progression following ASCT, there has been considerable interest in the development of postASCT consolidation and/or maintenance strategies which could lead to prolonged duration of disease control and improved survival. We review the data supporting the roles of both autologous and allogeneic transplant as well as posttransplant treatment strategies.

\section{Autologous stem cell transplant}

After induction therapy, consolidation consisting of high-dose melphalan with stem cell support has been a standard of care following induction therapy for newly diagnosed myeloma patients for decades. Transplant eligibility is often based on factors such as age and comorbidities. In some countries, ASCT is typically not offered for patients over the age of 65 years, however, in the United States, many transplant centers routinely transplant patients in the eighth decade without a strict age limit. Retrospective analyses have demonstrated the feasibility of performing ASCT in older adults based on performance status without inferior survival rates. ${ }^{2}$

Two studies performed prior to the development of immunomodulatory drugs (IMiDs) and proteasome inhibitors (PIs) revealed improved progression-free survival (PFS) and overall survival (OS) for patients who underwent ASCT as opposed to low-dose chemotherapy. . $^{3,4}$ However, the question of whether high-dose melphalan with stem cell support continues to be necessary following induction therapy in today's era of effective novel agents remains an active area of research (Table 1). In the melphalan $200 \mathrm{mg} / \mathrm{m}^{2}$ (MEL200) vs melphalan, prednisone, lenalidomide (MPR) study, patients received lenalidomide/ dexamethasone $(\mathrm{Rd})$ induction therapy followed by randomization to either tandem ASCT or to 6 cycles of oral MPR. Significant improvements in both PFS and OS were observed with the ASCT arm. ${ }^{5}$ In a similar study by the same group, patients were randomized to ASCT vs 6 cycles of oral cyclophosphamide, lenalidomide, dexamethasone (CRD) following Rd induction. ${ }^{6}$ This study also showed both a PFS and OS benefit for the ASCT arm. In both studies, benefit was observed for the ASCT arm regardless of cytogenetic risk group. These studies did not contain a PI as part of induction and/or consolidation. Triplet induction therapy, most commonly consisting of an IMiD and PI, has become a standard (eg, lenalidomide, bortezomib, dexamethasone [RVD]). The Intergroupe Francophone du Myélome (IFM)/Dana-Farber Cancer Institute (DFCI) 2009 study treated 
Table 1. Recent randomized studies comparing ASCT to conventional chemotherapy

\begin{tabular}{|c|c|c|c|c|c|c|}
\hline Study & Induction & $\begin{array}{c}\text { ASCT/ } \\
\text { Consolidation }\end{array}$ & Maintenance $^{*}$ & PFS & os & $\begin{array}{l}\text { Salvage ASCT } \\
\text { at relapset }\end{array}$ \\
\hline $\begin{array}{l}\text { GIMEMA } \\
\text { RV- } \\
209^{5}\end{array}$ & $\mathrm{Rd} \times 4$ & $\begin{array}{l}\text { Tandem ASCT vs } \\
\text { MPR } \times 6\end{array}$ & $\begin{array}{l}\text { Each arm randomized to } \\
\text { Len vs no maintenance }\end{array}$ & $\begin{array}{l}\text { Median PFS: } 43.0 \mathrm{mo} \\
\text { (MEL200) vs } 22.4 \mathrm{mo} \\
\text { (MPR) }(P<.001)\end{array}$ & $\begin{array}{l}\text { 4-y OS: } 81.6 \% \text { (MEL200) } \\
\text { vs } 65.3 \%(\mathrm{MPR}) \\
(P=.02)\end{array}$ & $\begin{array}{l}6 \%(\text { MEL200) } \\
\text { vs } 70 \% \text { (MPR) }\end{array}$ \\
\hline $\begin{array}{l}\text { RV-MM- } \\
\text { EMN- } \\
441^{6}\end{array}$ & $\mathrm{Rd} \times 4$ & $\begin{array}{l}1 \text { or } 2 \text { ASCT vs } \\
\text { CRD } \times 6\end{array}$ & $\begin{array}{l}\text { Each arm randomized to } \\
\text { Len alone vs Len plus } \\
\text { prednisone until } \\
\text { progression }\end{array}$ & $\begin{array}{l}\text { Median PFS: } 43.3 \mathrm{mo} \\
\text { (MEL200) vs } 28.6 \mathrm{mo} \\
\text { (CRD) }(P<.001)\end{array}$ & $\begin{array}{l}\text { 4-y OS: } 86 \% \text { (MEL200) } \\
\text { vs } 73 \%(\mathrm{CRD}) \\
(P=.004)\end{array}$ & $\begin{array}{l}21 \% \text { (MEL200) } \\
\text { vs } 43 \% \text { (CRD) }\end{array}$ \\
\hline $\begin{array}{l}\text { IFM/DFCl } \\
2009^{7}\end{array}$ & RVD $\times 3$ & $\begin{array}{l}\text { ASCT followed by } \\
\text { RVD } \times 2 \text { vs } \\
\text { RVD } \times 5\end{array}$ & $\begin{array}{l}\text { Len for both arms until } \\
\text { progression (US) or for } 1 \mathrm{y} \\
\text { (French) }\end{array}$ & $\begin{array}{l}\text { 3-y PFS } \ddagger \text { : } 61 \% \text { (ASCT) } \\
\text { vs } 48 \% \text { (RVD) }\end{array}$ & $\begin{array}{l}\text { 3-y OS } \neq: 88 \% \text { in both } \\
\text { arms }\end{array}$ & Not reported \\
\hline $\begin{array}{l}\mathrm{EMNO}^{2 /} \\
\mathrm{HO}^{8} 5^{8}\end{array}$ & $\begin{array}{l}\text { VCD } \times 3- \\
\quad 4\end{array}$ & $\begin{array}{l}\text { First randomization: } \\
\text { ASCT ( } 1 \text { or } 2) \\
\text { vs VMP } \\
\text { Second } \\
\text { randomization: } \\
\text { VRD } \times 2 \text { vs none }\end{array}$ & $\begin{array}{l}\text { Len for both arms until } \\
\text { progression }\end{array}$ & $\begin{array}{l}\text { 3-y PFS: } 66 \% \text { (ASCT) vs } \\
57.5 \% \text { (VMP) }\end{array}$ & $\begin{array}{l}\text { No differences but values } \\
\text { not yet reported }\end{array}$ & Not reported \\
\hline
\end{tabular}

Len, lenalidomide.

*Len was dosed 21 of 28 days in all studies except the IFM/DFCI 2009 study where it was dosed daily.

†Percentage of patients who relapsed while in the maintenance phase who received salvage ASCT.

‡Results are for the IFM study; US study still accruing.

transplant-eligible patients with 3 cycles of RVD induction followed by cyclophosphamide mobilization and stem cell collection. Patients are randomized to upfront vs delayed transplant. In the upfront arm, patients undergo ASCT followed by 2 cycles of RVD consolidation and then lenalidomide maintenance. In the delayed arm, after stem cell collection, patients complete 5 additional cycles of RVD consolidation followed by lenalidomide maintenance. Of note, the IFM stops lenalidomide maintenance after 1 year whereas in the United States, lenalidomide is continued until progression. The preliminary results from the IFM show a PFS benefit for upfront ASCT with no difference in OS. ${ }^{7}$ The initial results from the European Myeloma Network/Stichting HematoOncologie voor Volwassenen Nederland (HOVON) EMN02/HO95 were recently reported. ${ }^{8}$ In this study, patients received induction therapy with bortezomib, cyclophosphamide, dexamethasone (VCD) followed by randomization to ASCT (1 or 2) vs bortezomib, melphalan, prednisone (VMP). A second randomization includes further consolidation with bortezomib, lenalidomide, dexamethasone (VRD) vs observation. All patients go on to receive lenalidomide maintenance. The 3-year PFS favors the ASCT arm (66\% vs 57.5\%; hazard ratio [HR], 0.73; $P=.003$ ). In aggregate, these studies demonstrate a PFS benefit with early ASCT, confirming the continued role of ASCT.

- Outside of the context of a clinical trial, we recommend the use of ASCT following induction therapy for patients who are transplant-eligible.

These 4 studies (Table 1) did not directly address the role of ASCT at time of first relapse. It is assumed that patients who progress in the nontransplant or delayed arm will be offered ASCT as second-line therapy, but this is not mandated by the studies. In the MEL200 vs MPR study, $70 \%$ of patients underwent ASCT as part of second-line therapy after relapse whereas in the MEL200 vs CRD study, $43 \%$ of patients on the CRD arm received ASCT as second-line therapy. ${ }^{5,6}$ Notably, in a pooled analysis of these 2 studies, the patients who received conventional chemotherapy plus lenalidomide maintenance had improved OS (HR, 0.57; $P=.037$ ) if they underwent ASCT at time of relapse compared with those who did not undergo ASCT, ${ }^{9}$ providing further evidence for the benefit of ASCT.

- Transplant-eligible patients who do not undergo upfront ASCT should be offered ASCT at time of first relapse.

\section{Tandem ASCT}

The use of planned sequential ASCT, or "tandem" ASCT, was first introduced by the University of Arkansas ${ }^{10}$ and continues to form the backbone of the "total therapy" approach. Whether tandem ASCT produces superior outcomes compared with single ASCT has been the focus of several randomized studies (Table 2). Meta-analyses have revealed superior response rates with tandem ASCT with no difference in OS and were limited due to study heterogeneity. ${ }^{11,12}$ More recently, long-term follow-up of the German-Speaking Myeloma Multicenter Group (GMMG)-HD2 study was reported. With a median follow-up of 11 years, single ASCT was shown to be noninferior to tandem ASCT with no differences in either event-free survival (EFS) or OS. ${ }^{13}$ Results are pending for the Blood and Marrow Transplant Clinical Trials Network (BMT CTN) 0702 study which randomizes patients to 1 of 3 arms: single ASCT with lenalidomide maintenance, single ASCT with RVD consolidation and lenalidomide maintenance, and tandem ASCT with lenalidomide maintenance (NCT01109004). Of interest is whether a particular risk group (cytogenetic, International Scoring System [ISS], or minimal residual disease [MRD] positivity) might benefit more from one approach compared with another.

\section{Salvage ASCT}

There have been a number of reports documenting the feasibility of performing salvage ASCT. Overall response rates are generally high (55\%-97\%) and transplant-related mortality rates low (2\%-8\%) (Reviewed in Holstein et $\mathrm{al}^{14}$ ). Overall, the PFS benefit from a salvage transplant is approximately half the PFS from the first transplant. There is no clear signal that the type of high-dose therapy given with the salvage ASCT has a significant impact on outcome. In the absence of prospective trial data, many centers continue to use single-agent high-dose melphalan. Incorporation of agents such as busulfan or bortezomib into the highdose melphalan regimens have not clearly generated superior results for upfront or salvage ASCT. Patients who relapse early after the first ASCT derive less benefit from salvage ASCT and have worse outcomes. A Center for International Blood and Marrow Transplant Research (CIBMTR) analysis of 187 patients who underwent salvage ASCT demonstrated that patients who relapsed 36 months or more after the first 


\begin{tabular}{|c|c|c|c|c|c|c|}
\hline Study & First ASCT & $\begin{array}{l}\text { Consolidation } s / p \\
\text { first ASCT }\end{array}$ & Maintenance & EFS & os & $\begin{array}{l}\text { Salvage ASCT } \\
\text { at relapse }\end{array}$ \\
\hline IFM94 54 & $\begin{array}{l}\text { MEL140 + } \\
\text { TBI (single } \\
\text { arm) } \\
\text { MEL140 } \\
\text { (tandem } \\
\text { arm) }\end{array}$ & None vs MEL140 + TBI & Interferon $\alpha$ & $\begin{array}{l}\text { Median EFS: } 25 \\
\quad \text { (single) vs } 30 \\
\text { (tandem) mo } \\
(P=.03)\end{array}$ & $\begin{array}{l}\text { Median OS: } 48 \text { (single) vs } \\
58 \text { (tandem) mo }(P= \\
.01)\end{array}$ & $\begin{array}{l}22 \% \text { (single arm) } \\
\text { vs } 26 \% \text { (tandem } \\
\text { arm) }\end{array}$ \\
\hline $\begin{array}{c}\text { Bologna } \\
76^{55}\end{array}$ & MEL200 & $\begin{array}{l}\text { None vs MEL120 + } \\
\text { busulfan }\end{array}$ & Interferon $\alpha$ & $\begin{array}{l}\text { Median EFS: } 23 \\
\quad \text { (single) vs } 25 \\
\text { (tandem) mo } \\
(P=.001)\end{array}$ & $\begin{array}{l}7-y \text { OS: } 46 \% \text { (single) vs } \\
43 \% \text { (tandem) }(P=.9)\end{array}$ & $\begin{array}{l}33 \% \text { (single arm) } \\
\text { vs } 10 \% \text { (tandem } \\
\text { arm) }\end{array}$ \\
\hline $\begin{array}{l}\text { GMMG- } \\
\qquad D 2^{13}\end{array}$ & MEL200 & $\begin{array}{l}\text { None vs second ASCT } \\
\text { with MEL200 }\end{array}$ & Interferon $\alpha$ & $\begin{array}{l}\text { Median EFS: } 25 \\
\text { (single) vs } 28.7 \\
\text { (tandem) mo } \\
(P=.53)\end{array}$ & $\begin{array}{l}\text { Median OS: } 73 \text { (single) vs } \\
75.3 \text { (tandem) mo } \\
(P=.33)\end{array}$ & $\begin{array}{l}26 \% \text { (single arm) } \\
\text { vs } 24 \% \text { (tandem } \\
\text { arm) }\end{array}$ \\
\hline $\begin{array}{l}\text { BMT } \\
\text { CTN } \\
\text { 0702 } \\
\text { (NCT01109004) }\end{array}$ & MEL200 & $\begin{array}{l}\text { None vs RVD } \times 4 \text { vs } \\
\text { second ASCT with } \\
\text { MEL200 }\end{array}$ & $\begin{array}{l}\text { Len until } \\
\text { progression } \\
\text { for all arms }\end{array}$ & Not yet reported & Not yet reported & Not yet reported \\
\hline
\end{tabular}

s/p, status-post; TBI, total body irradiation.

transplant derived greater benefit from the second transplant (longer PFS and OS) than those who relapsed early. ${ }^{15}$ Where the cutoff should be for relapse from first ASCT for considering patients for salvage ASCT has been a matter of debate, but a recent workshop generated consensus guidelines that patients relapsing after $>18$ months from their first ASCT should be considered for salvage ASCT. ${ }^{16}$ When salvage transplant has been compared with salvage chemotherapy following relapse from first ASCT, retrospective analyses have shown trends toward improved OS. ${ }^{14}$ A multicenter, randomized phase 3 study compared salvage ASCT with cyclophosphamide ( $400 \mathrm{mg} / \mathrm{m}^{2}$ per week for 12 weeks). ${ }^{17}$ Patients were eligible if they had progressed or relapsed at least 18 months after the initial ASCT. All patients received reinduction with bortezomibdoxorubicin-dexamethasone and were randomized to either ASCT or cyclophosphamide. The median time to progression was longer in the ASCT arm (19vs 11 months; $P<.0001)$ and the median OS was superior in the salvage ASCT arm compared with the weekly cyclophosphamide arm (67 vs 52 months; $P=.0169)$. There are now salvage consolidation regimens that are likely more effective than single-agent cyclophosphamide. Prospective randomized trials comparing novel therapies (eg, pomalidomide-carfilzomib-dexamethasone, or other novel agent combinations including monoclonal antibodies) with salvage ASCT are needed.

- We recommend salvage ASCT for patients who remain transplant eligible and who achieved at least 18 months of disease control following initial ASCT.

\section{AlloSCT}

Allogeneic stem cell transplant (AlloSCT) with myeloablative conditioning for myeloma has historically been associated with high transplant-related mortality (TRM) rates in the $40 \%$ to $60 \%$ range (reviewed in Dhakal et $\mathrm{al}^{18}$ ). Therefore, more recent efforts have focused on the development of nonmyeloablative or reducedintensity conditioning (RIC) approaches. ${ }^{18}$ In the upfront setting, there have been several studies which have compared tandem ASCTAlloSCT to tandem ASCT but results of these studies have not been consistent (reviewed in Dhakal et $\mathrm{al}^{18}$ ). For example, although studies such as BMT CTN $0102^{19}$ and IFM9903-04 ${ }^{20}$ did not show an OS benefit for AlloSCT, studies such as those of Bruno et $\mathrm{al}^{21}$ and the European Group for Blood and Marrow Transplantation-
Non-Myeloablative Allogeneic Stem Cell Transplantation in Multiple Myeloma (EBMT-NMAM) ${ }^{22}$ demonstrated an OS benefit, albeit in some cases only in the high-risk subsets. Prospective randomized studies comparing salvage AlloSCT with salvage ASCT have not been performed. In aggregate, the retrospective studies comparing these 2 approaches have revealed a higher nonrelapse mortality rate with AlloSCT with median OS favoring ASCT, but it should be noted that they are limited by lack of information regarding risk status from cytogenetic data. ${ }^{14}$ RIC AlloSCT in the salvage setting can be considered for younger patients with good performance status and high-risk features such as del $17 \mathrm{p}, \mathrm{t}(4 ; 14), \mathrm{t}(14 ; 16)$, or high-risk gene expression profile, high lactate dehydrogenase, or plasma cell leukemia. Patients with high volume disease do not respond well to AlloSCT, and disease control prior to the AlloSCT is necessary for long-term disease control. The role of RIC AlloSCT in the upfront setting is less clear but can be considered for the very-high-risk patient and, if possible, in the context of a clinical trial. The BMTCTN is studying RIC AlloSCT for high-risk disease with a randomization to ixazomib vs placebo after transplant (NCT02440464).

\section{Consolidation following ASCT}

Consolidation following ASCT commonly involves a short course of either single-agent or multiagent therapy. The intent of consolidation is to improve the depth of response with the expectation this will improve long-term outcomes. Randomized studies involving postASCT consolidation approaches are summarized in Table 3 and, in general, although consolidation does appear to improve response rates, there has not been strong evidence that this translates to improved OS. Cavo et al compared thalidomide/dexamethasone (TD) to TD plus bortezomib (VTD) as induction therapy followed by tandem ASCT and then 2 cycles of TD or VTD consolidation. ${ }^{23}$ Although higher response rates were observed in the bortezomibcontaining arm, this arm was also associated with a higher incidence of adverse events, including peripheral neuropathy. The VTD arm had a higher PFS but no OS benefit was observed. The Nordic Myeloma Study Group performed a randomized study in which patients received no consolidation vs bortezomib consolidation. ${ }^{24}$ Although the PFS favored the bortezomib arm, there was no difference in OS. Straka et al recently reported the combined outcomes 


\begin{tabular}{|c|c|c|c|c|}
\hline Reference & Consolidation regimen & $\begin{array}{l}\text { Response rates } \\
\text { (pre- and post-) \% }\end{array}$ & PFS & os \\
\hline 23,56 & VTD vs TD & $\begin{array}{c}\text { nCR/CR rates } \\
\text { VTD: } 63 \rightarrow 73 \\
\text { TD: } 55 \rightarrow 61\end{array}$ & $\begin{array}{l}\text { 3-y PFS: } 60 \% \text { (VTD) vs } 48 \% \text { (TD) } \\
\quad(P=.042)\end{array}$ & $\begin{array}{l}\text { 3-y OS: } 90 \% \text { (VTD) vs } 88 \% \text { (TD) } \\
\quad(P=.39)\end{array}$ \\
\hline 24 & V vs none & $\begin{array}{l}\text { nCR/CR rates } \\
\text { V: } 20 \rightarrow 45 \\
\text { None: } 21 \rightarrow 35\end{array}$ & $\begin{array}{l}\text { Median PFS: } 27(\mathrm{~V}) \text { vs } 20 \text { (none) } \\
\quad \text { mo }(P=.05)\end{array}$ & Median OS not reached for either arm $(P=.4)$ \\
\hline 25 & V vs none & $\begin{array}{l}\geq \text { VGPR rates } \\
\text { V: } 55 \rightarrow 62 \\
\text { None: } 59 \rightarrow 48\end{array}$ & $\begin{array}{l}\text { Median PFS: } 33.6(\mathrm{~V}) \text { vs } 27.8 \\
\text { (none) mo }(P=.0058)\end{array}$ & Median OS not reached for either arm $(P=.75)$ \\
\hline
\end{tabular}

V, bortezomib; VGPR, very-good-partial response.

of 2 randomized studies which compared bortezomib consolidation vs observation post-ASCT. ${ }^{25}$ Again, although bortezomib improved response rates and PFS, no difference in OS was found. ${ }^{25}$ The results of BMT-CTN 0702 should be informative with respect to the role of RVD consolidation following ASCT. It should be noted that improvement in response rates over time following ASCT (eg, the improvement in the placebo arm of the near complete response (nCR)/ complete response (CR) rate from $21 \%$ to $35 \%$ in the Mellqvist et al study $^{24}$ ) may be due to the half-life of the monoclonal immunoglobulins.

\section{Maintenance following ASCT}

Prior to the introduction of IMiDs and PIs, posttransplant maintenance strategies primarily involved interferon $\alpha$. Two meta-analyses demonstrate that the OS benefit achieved with interferon maintenance is small (3-4 months). ${ }^{26,27}$ The lack of prolonged benefit, the impact on quality of life due to the side-effect profile, and the development of the IMiDs and PIs has resulted in interferon being of interest only from a historical perspective.

There have been 8 randomized studies which have investigated the use of thalidomide following ASCT (for review, see McCarthy and Palumbo $^{28}$ ). These studies vary with respect to thalidomide dosing and inclusion or not of corticosteroids. Some trials demonstrated an improvement in OS with thalidomide whereas others did not. ${ }^{28}$ There is evidence that thalidomide maintenance does not improve outcomes for patients with adverse cytogenetics. ${ }^{29}$ Feasibility issues of prolonged thalidomide therapy due to toxicity (particularly peripheral neuropathy), have limited the median duration of maintenance therapy to $\sim 1$ year. Given the more favorable side-effect profile of lenalidomide with respect to neurotoxic effects, the use of thalidomide in the posttransplant setting has significantly diminished in the United States. However, thalidomide maintenance remains an option, especially when combined with glucocorticoids, for patients who cannot tolerate the myelosuppressive effects of lenalidomide. It is a more cost-effective option in areas with limited access to lenalidomide or bortezomib.

The use of lenalidomide as maintenance therapy following ASCT has been studied in 3 randomized studies, all demonstrating a PFS benefit with varying effects on OS (Table 4). A recent meta-analysis of these 3 studies found that lenalidomide maintenance significantly improves OS, regardless of response achieved post-ASCT. ${ }^{30}$ Cancer and Leukemia Group B (CALGB) 100104, a phase 3 study, randomized 460 patients to receive lenalidomide vs placebo following ASCT. ${ }^{31}$ The study was unblinded after interim analyses demonstrated that the primary end point of time to progression (TTP) was met (46 months vs 27 months; HR $=0.48 ; P<.001$ ). At the time of unblinding, 86 of 128 nonprogressing patients on the placebo arm chose to cross over and receive lenalidomide. At 34 months median follow-up, there was an OS benefit for lenalidomide (85\% vs $77 \%$; $P=.03)$. An update with a 65-month median follow-up for OS continues to show substantial improvements in both TTP (53 vs 27 months; $\mathrm{HR}=0.54 ; P<.0001)$ and OS (median OS not reached for lenalidomide vs 76 months for placebo; $P=.001) .{ }^{32}$ No difference in OS after disease progression was observed between the 2 arms. The IFM 2005-02 study included 614 patients randomized to receive lenalidomide vs placebo maintenance following 2 cycles of lenalidomide consolidation therapy (25 mg per day for days $1-21$ ) post-ASCT. ${ }^{33}$ This study was unblinded once the primary end point of PFS was met (median PFS of 41 months vs 23 months; HR, $0.5 ; P<.001$ ). There was no crossover to lenalidomide. Maintenance therapy was discontinued after 1 to 3 years (median, 2 years) due to a concern about the development of second primary malignancies (SPMs). At 60 months postrandomization, there continues to be a PFS benefit for lenalidomide (42\% vs $18 \%$; $P<.0001)$ and equivalent 5 -year OS rates $(68 \%$ vs $67 \%) .{ }^{34}$ Both OS after progression and median second PFS (defined as time from progression in first-line to second progression or death) were inferior on the lenalidomide arm. None of the patients in the IFM 2005-02 study received IMiD-containing induction therapy whereas in CALGB 100104, $35 \%$ of patients received a lenalidomide-containing regimen and $45 \%$ of patients received a thalidomide-containing regimen. To be eligible for CALGB 100104, patients could not have progressed while receiving induction therapy. The E4A03 study ${ }^{35}$ demonstrated that the percentages of patients on lenalidomide-dexamethasone induction who either progressed or had no response/stable disease were $3 \%$ and $6 \%$, respectively, thus it is unlikely that CALGB 100104 was overweighted with lenalidomide-sensitive patients compared with IFM 05-02.

The Gruppo Italiano Malattie EMatologiche dell'Adulto (GIMEMA) RV-209 (MPR vs MEL200) study also evaluated the role of lenalidomide maintenance therapy after low-dose therapy or ASCT. ${ }^{5}$ An analysis combining the tandem ASCT and MPR arms revealed that lenalidomide maintenance improved median PFS (42 months) compared with no maintenance (22 months) $(\mathrm{HR}=0.47 ; P<.001)$. There was a trend toward improved 3-year OS rates with lenalidomide maintenance (88 vs $79 \% ; P=.14$ ). The GIMEMA CRD vs tandem MEL200 study randomized patients to lenalidomide vs lenalidomide and prednisone maintenance. ${ }^{6}$ As with the preceding MPR vs MEL200 study, the reported analysis combined the ASCT and chemotherapy arms to assess the impact of the addition of prednisone to lenalidomide maintenance. No difference was observed in PFS between the 2 maintenance arms. ${ }^{6}$

The most common adverse events associated with lenalidomide maintenance therapy have been hematological in nature. In the CALGB 100104 study, 69\% of patients in the lenalidomide arm and 


\begin{tabular}{|c|c|c|c|c|c|c|}
\hline Study & $\mathbf{n}$ & $\begin{array}{l}\text { Induction } \\
\text { therapy }\end{array}$ & $\begin{array}{l}\text { Dosing } \\
\text { schedule }\end{array}$ & $\begin{array}{c}\text { Duration of } \\
\text { maintenance }\end{array}$ & $\begin{array}{l}\text { EFS or PFS } \\
\text { (maintenance } \\
\text { vs no) }\end{array}$ & $\begin{array}{c}\text { OS (maintenance } \\
\text { vs no) }\end{array}$ \\
\hline $\begin{array}{l}\text { CALGB } \\
\qquad 100104^{31,32}\end{array}$ & 460 & $\begin{array}{l}\leq 2 \text { regimens; } 94 \% \\
\text { received a regimen } \\
\text { containing Thal, Len, } \\
\text { and/or Bor }\end{array}$ & $\begin{array}{l}10 \mathrm{mg} \text { continuous, } \\
\text { increase up to } 15 \mathrm{mg}\end{array}$ & Until progression & $\begin{array}{l}\text { Median TTP: } 53 \text { vs } \\
\quad 27 \text { mo }(P<.001)\end{array}$ & $\begin{array}{l}\text { Median follow-up of } \\
65 \text { mo: not reached } \\
\text { vs } 76 \text { mo }(P=.001)\end{array}$ \\
\hline $\begin{array}{l}\text { IFM } \\
\qquad 2005-02^{33,34}\end{array}$ & 614 & $\begin{array}{l}46 \% \text { received vincristine, } \\
\text { doxorubicin, Dex and } \\
46 \% \text { received Bor } \\
\text { and Dex } \\
21 \% \text { received tandem } \\
\text { transplant } \\
25 \% \text { received induction } \\
\text { reinforcement with DCEP }\end{array}$ & $\begin{array}{l}\text { All patients received } \\
2 \text { cycles of } \\
\text { consolidation } \\
(25 \mathrm{mg} / \mathrm{d}, 21 \text { of } 28 \mathrm{~d}) \\
\text { Maintenance: } 10 \mathrm{mg} \\
\text { continuous, increase } \\
\text { up to } 15 \mathrm{mg}\end{array}$ & $\begin{array}{l}\text { Stopped due to } \\
\text { concerns regarding } \\
\text { second primary } \\
\text { malignancies at a } \\
\text { median time of } 2 \mathrm{y} \\
\text { (range, } 1-3 \mathrm{y} \text { ) }\end{array}$ & $\begin{array}{l}\text { 4-y PFS: } 43 \text { vs } \\
22 \%(P<.001) \\
\text { 5-y PFS: } 42 \% \text { vs } \\
18 \%(P<.001)\end{array}$ & $\begin{array}{l}\text { Median follow-up } \\
\quad 45 \text { mo: } 74 \text { vs } \\
76 \%(P=.7) \\
\text { 4-y OS: } 73 \% \\
\quad \text { vs } 75 \%(P=.7) ; 5 \text {-y } \\
\text { OS: } 68 \% \text { vs } 67 \% \\
\text { (HR }=1)\end{array}$ \\
\hline $\begin{array}{l}\text { GIMEMA } \\
\text { RV-209 }\end{array}$ & 402 & $\begin{array}{l}4 \text { cycles Len/Dex } \\
\text { followed by either } \\
\text { tandem transplant } \\
\text { (MEL200) or MPR }\end{array}$ & $\begin{array}{c}10 \mathrm{mg} \text { (3 wk on, } \\
1 \mathrm{wk} \text { off) }\end{array}$ & Until progression & $\begin{array}{r}\text { Median PFS*: } 42 \text { vs } \\
22 \text { mo }(P<.001)\end{array}$ & $\begin{array}{l}\text { 3-y OS*: } 88 \% \\
\quad \text { vs } 79 \%(P=.14)\end{array}$ \\
\hline
\end{tabular}

Bor, bortezomib; DCEP, dexamethasone, cyclophosphamide, etoposide, cisplatin; Dex, dexamethasone; Thal, thalidomide.

${ }^{\star}$ Combining MEL200 and MPR groups.

$30 \%$ of the placebo arm patients developed grade $3 / 4$ adverse events, of which $48 \%$ and $17 \%$, respectively, were hematologic. ${ }^{31}$ Ten percent of patients on the lenalidomide arm stopped therapy due to adverse events compared with $1 \%$ on the placebo arm and $6 \%$ of patients who crossed over to receive lenalidomide. In the IFM 2005-02 study, $74 \%$ of patients receiving lenalidomide and $43 \%$ receiving placebo had grade 3 or 4 events, of which $58 \%$ and $22 \%$, respectively, were hematologic. ${ }^{33}$ The discontinuation rates on this study were $27 \%$ (lenalidomide arm) and 15\% (placebo arm).

An increased incidence of SPMs in patients receiving lenalidomide maintenance has been reported in both the CALGB and IFM studies. The most recent update of the IFM study has reported a total of $20(6.6 \%)$ hematological malignancies and $24(7.8 \%)$ solid tumors in 35 patients in the lenalidomide arm and $6(1.9 \%)$ hematological malignancies and $22(7.2 \%)$ solid tumors in 20 patients in the placebo arm. ${ }^{34}$ With the updated analysis of CALGB 100104, there have now been 14 (6.1\%) hematological and $11(4.8 \%)$ solid tumors in the lenalidomide arm compared with $3(1.3 \%)$ hematological and $7(3.1 \%)$ solid tumors in the placebo arm. ${ }^{32}$ The cumulative incidence risk of developing a SPM is higher for lenalidomide compared with placebo $(P=.005)$ and the cumulative incidence risk of disease progression $(P<.001)$ or death $(P<.001)$ is higher for placebo. The majority of hematological SPMs reported in these 2 studies have been myeloid in nature (myelodysplastic syndrome or acute myeloid leukemia). However, there have also been B-cell acute lymphoblastic leukemia (ALL) SPMs (5 on CALGB 100104 and 3 on IFM 05-02). It is unknown whether the risk factors for developing ALL on lenalidomide post-ASCT are different from those contributing to myelodysplastic syndrome/acute myeloid leukemia. Of note, deletions in the gene for the transcription factor IKZF1, which is degraded following IMiD binding to cereblon, have been associated with B-cell ALL. ${ }^{36}$ Additional studies are needed to determine the mechanisms underlying the association between lenalidomide maintenance therapy and development of ALL as an SPM and SPMs in general.

- Lenalidomide maintenance post-ASCT continued until progression is a standard of care. Patients should be monitored carefully as there is a risk for the development of second primary malignancies.
Unlike lenalidomide, there have not been any placebo-controlled randomized studies conducted with bortezomib in the post-ASCT setting. The study most frequently cited to support the use of bortezomib maintenance is the HOVON-65/GMMG-HD4 trial. ${ }^{37}$ This study involved 827 newly diagnosed patients randomized to receive either vincristine, doxorubicin, dexamethasone (VAD) or bortezomib, doxorubicin, dexamethasone (PAD) induction therapy followed by either single or tandem ASCT. Patients on the VAD arm received thalidomide maintenance whereas those on the PAD arm received bortezomib maintenance given every other week for 2 years. The study design did not allow for direct comparison of the 2 maintenance regimens. However, bortezomib maintenance following a bortezomib-containing induction improved the $\mathrm{nCR}$ plus $\mathrm{CR}$ rate and was better tolerated than thalidomide maintenance after VAD induction. When PFS was calculated from time of ASCT, a statistically significant benefit was seen with bortezomib. When adjusted for the ISS, the OS was superior on the PAD arm $(\mathrm{HR}=0.80 ; P=.047)$ and the PAD arm also demonstrated improved PFS and OS for patients in renal failure. A recent update with a median follow-up of 91.4 months showed a persistent improvement in both PFS and OS for those patients who received bortezomib induction/maintenance. ${ }^{38}$ This study forms the basis for using every-other-week bortezomib as post-ASCT maintenance therapy. The prolonged use of bortezomib can be limited by the development or worsening of existing peripheral neuropathy. Data have not emerged that bortezomib maintenance is associated with SPMs.

\section{Adverse-risk cytogenetics and post-ASCT therapy}

Despite overall improvements in outcomes for patients with myeloma, those with adverse-risk cytogenetics such as del(17p), monosomy $13, \mathrm{t}(4 ; 14)$, and $\mathrm{t}(14 ; 16)$ continue to have inferior outcomes. Multiple reports have suggested that bortezomib may partially overcome the adverse prognosis associated with $t(4 ; 14)$ and chromosome 13 deletion. In the HOVON trial, bortezomib significantly decreased the risk associated with del(17p) whereas there was no significant difference in outcomes for patients with $\mathrm{t}(4 ; 14)$ or $\operatorname{add}(1 \mathrm{q}) .{ }^{37}$ For lenalidomide maintenance, CALGB 100104 did not report outcomes based on cytogenetics ${ }^{31}$ whereas the IFM 2005-02 study reported that the HR for progression or death favored the 
Table 5. Ongoing phase 3 consolidation/maintenance studies incorporating novel agents

\begin{tabular}{|c|c|c|c|c|}
\hline Study & Induction & Consolidation s/p ASCT & Maintenance & Primary end point \\
\hline NCT02181413 & Not specified & None & Ixa vs placebo for $2 \mathrm{y}$ & PFS \\
\hline SWOG (planned) & Not specified & None & Len + Ixa vs Len until PD & os \\
\hline NCT02406144 & $\begin{array}{l}\text { RVD } \times 6 \text { (patients on } \\
\text { GEM2012MENOS65 } \\
\text { (NCT01916252), } \\
\text { randomized to MEL200 vs } \\
\text { BUMEL for ASCT high- } \\
\text { dose therapy }\end{array}$ & $\begin{array}{l}\text { RVD } \times 2 \text { (patients on } \\
\text { GEM2012MENOS65) }\end{array}$ & $\begin{array}{l}\text { Len }+ \text { Dex for } 2 \text { y or Len } \\
+ \text { Dex }+ \text { Ixa for } 2 \text { y } \\
\text { At } 2 \text { y, MRD- stop } \\
\text { maintenance, MRD+ } \\
\text { continue Len + Dex } \\
\text { for } 3 y\end{array}$ & PFS \\
\hline $\begin{array}{l}\text { GMMG-HD6 } \\
\text { (NCT02495922) }\end{array}$ & $\begin{array}{l}\text { VRD } \times 4 \\
\text { VRD } \times 4 \\
\text { VRD }+ \text { Elo } \times 4 \\
\text { VRD }+ \text { Elo } \times 4\end{array}$ & $\begin{array}{l}\text { VRD } \times 2 \\
\text { VRD }+ \text { Elo } \times 2 \\
\text { VRD } \times 2 \\
\text { VRD }+ \text { Elo } \times 2\end{array}$ & $\begin{array}{l}\text { Len }+ \text { Dex for } 2 y \\
\text { Len }+ \text { Dex }+ \text { Elo for } 2 y \\
\text { Len }+ \text { Dex for } 2 y \\
\text { Len }+ \text { Dex }+ \text { Elo for } 2 y\end{array}$ & PFS \\
\hline $\begin{array}{l}\text { DSMM XIV } \\
\quad \text { (NCT01685814) }\end{array}$ & RAD $\times 3$ vs VRD $\times 3$ & $\begin{aligned} \text { If } & \geq \text { VGPR after first } A S C T, \\
& \text { randomization to nothing } \\
& \text { vs second } A S C T . \\
\text { If } & <\text { VGPR after first } A S C T, \\
& \text { randomization to second } \\
& \text { ASCT vs AlloSCT }\end{aligned}$ & $\begin{array}{l}\text { For single/tandem } \\
\text { ASCT: Len for } 3 \text { y } \\
\text { For AlloSCT: Len for } 1 \mathrm{y}\end{array}$ & $\begin{array}{l}\text { Induction phase: CR rate } \\
\text { Consolidation phase: PFS }\end{array}$ \\
\hline $\begin{array}{l}\text { FORTE } \\
\text { (NCT02203643) }\end{array}$ & $\begin{array}{l}\text { KCd } \times 4 \text { then } \mathrm{ASCT} \\
\mathrm{KRd} \times 4 \text { then } \mathrm{ASCT} \\
\mathrm{KCd} \times 4, \text { stem collection, } \\
\text { then KRd } \times 8\end{array}$ & $\begin{array}{l}\mathrm{KCd} \times 4 \\
\mathrm{KCd} \times 4\end{array}$ & $\begin{array}{l}\text { All arms randomized to } \\
\text { Len vs Len }+ \text { Car }\end{array}$ & $\geq$ VGPR rate after induction \\
\hline $\begin{array}{l}\text { MRC Myeloma XI + } \\
\quad(\text { NCT01554852) }\end{array}$ & $\begin{array}{l}\text { CRD vs CTD vs KRCD } \\
\text { VCD consolidation prior to } \\
\text { ASCT for }<\text { VGPR }\end{array}$ & None & $\begin{array}{l}\text { Len vs Len }+ \text { Vorinostat } \\
\text { vs Observation }\end{array}$ & $\begin{array}{l}\text { Induction: PFS/OS } \\
\text { Maintenance: PFS/OS }\end{array}$ \\
\hline $\begin{array}{l}\text { HOVON } 131 \mathrm{MM} / \mathrm{IFM} \\
\text { 2015-01 } \\
\text { (NCT02541383) }\end{array}$ & $\begin{array}{l}\text { VTD } \times 4 \\
\text { VTD-Dara } \times 4\end{array}$ & $\begin{array}{l}\text { VTD } \times 2 \\
\text { VTD-Dara } \times 2\end{array}$ & $\begin{array}{l}\text { Both arms randomized to } \\
\text { Dara vs observation }\end{array}$ & $\begin{array}{l}\text { sCR rate after consolidation } \\
\text { Maintenance: PFS/TTP }\end{array}$ \\
\hline
\end{tabular}

BUMEL, busulfan-melphalan; Car, carfilzomib; CRD, Cyclophosphamide, lenalidomide, dexamethasone; CTD, cyclophosphamide, thalidomide, dexamethasone; Dara, daratumumab; Elo, elotuzumab; Ixa, ixazomib; KCd, carfilzomib, cyclophosphamide, dexamethasone; KRCD, carfilzomib, lenalidomide, cyclophosphamide, dexamethasone; $\mathrm{KRd}$, carfilzomib, lenalidomide, dexamethasone; RAD, lenalidomide, adriamycin, dexamethasone; sCR, stringent CR; VRD, bortezomib, lenalidomide, dexamethasone (RVD). Other abbreviations are explained in Tables 1,3 , and 4.

lenalidomide arm for patients with $13 \mathrm{q}$ deletion, without $13 \mathrm{q}$ deletion, and without $\mathrm{t}(4 ; 14)$ or $17 \mathrm{p}$ deletion, but did not reach significance for patients with either $\mathrm{t}(4 ; 14)$ or $17 \mathrm{p}$ deletion. ${ }^{33}$ The management of highrisk myeloma is discussed in detail by Dispenzieri (in this book ${ }^{39}$ ).

\section{The role of ASCT for AL amyloidosis}

Although ASCT has been demonstrated to be capable of resulting in long-term survival for select amyloid light-chain (AL) patients, the routine use of ASCT for this disease has been complicated by the fact that many patients are ineligible or "unfit" for ASCT because of severe organ involvement (particularly cardiac) as well as the increased morbidity and mortality associated with the transplant procedure (reviews, Sher et $\mathrm{al}^{40}$ and Palladini and $\mathrm{Merlini}^{41}$ ). One randomized study has been performed comparing ASCT to standard chemotherapy (melphalan-dexamethasone) in patients with AL amyloidosis. ${ }^{42}$ This study involved 100 newly diagnosed AL amyloid patients. The median OS was 22.2 months in the ASCT group and 56.9 months in the melphalan-dexamethasone group $(P=.04)$. Notably, the TRM rate was $24 \%$. More recently, better patient selection and improved supportivecare strategies have led to reductions in TRM. ${ }^{40,41}$ Whether the outcomes of ASCT can be further improved with the incorporation of PI therapy is an active area of research. Furthermore, it is tempting to speculate that the monoclonal antibody NEOD001, which targets misfolded proteins and induces organ responses in AL amyloidosis, ${ }^{43}$ could be used as maintenance therapy following ASCT to enhance long-term outcomes.

The question of whether a lower dose of melphalan with ASCT results in lower TRM was assessed in the SWOG-0115 study. ${ }^{44}$ This study enrolled patients with AL amyloid, AL amyloid with myeloma, or high-risk myeloma (defined as older patients with renal failure) and included 2 cycles of modified high-dose melphalan (MEL100 mg/m²) with ASCT. The overall TRM rate was $12 \%$ with $8 \%$ occurring during stem mobilization/collection and $4 \%$ occurring within the first 100 days after the second ASCT. Only 56\% of the AL patients received the second ASCT. The median OS for the AL group was 5.7 years.

Currently, there are not data to support the use of post-ASCT maintenance therapy for AL amyloidosis. There are retrospective data demonstrating that doxycycline as part of standard chemotherapy ${ }^{45}$ and following transplant ${ }^{46}$ improves outcome in cardiac amyloidosis. As bortezomib has been shown to induce rapid hematologic responses as well as organ responses in $\mathrm{AL}$ amyloidosis, ${ }^{47}$ its use has been studied in the pre- and peri-ASCT setting. ${ }^{48,49}$ It remains to be determined whether incorporation of bortezomib (or other PIs), IMiDs, monoclonal antibodies, or other agents can improve outcomes post-ASCT without increasing toxicity.

- ASCT is an option for fit AL amyloidosis patients based on phase 2 data. Future studies of risk stratification and incorporation of newer therapeutic agents are necessary.

\section{Future directions}

In 2015, 4 new drugs were approved for relapsed/refractory myeloma including the pan-histone deacetylase inhibitor panobinostat, the first oral PI ixazomib, a monoclonal antibody against CD38 (daratumumab), and a monoclonal antibody against SLAMF7 (elotuzumab). There are multiple ongoing or planned phase 3 studies which are incorporating some of these various agents into the post-ASCT setting (Table 5). 
Unlike the currently available PIs, ixazomib is an oral agent and can be administered on a weekly basis. Although chemically similar to bortezomib, ixazomib is associated with less peripheral neuropathy and is a reasonable candidate for prolonged maintenance therapy. A previously reported study demonstrated the feasibility of ixazomib maintenance therapy for up to 1.5 years following ixazomib-lenalidomide-dexamethasone induction therapy. ${ }^{50}$ Adverse events of ixazomib maintenance included diarrhea, nausea, and cytopenias but with minimal peripheral neuropathy. Although CALGB 100104 supports continuation of lenalidomide until disease progression, it is not known whether ixazomib should be similarly continued until progression and there are varying durations of treatment in the ongoing and planned studies. It will be of particular interest to determine whether ixazomib, especially in combination with other agents such as lenalidomide, can overcome poor-risk cytogenetics. Studies performed thus far have demonstrated acceptable side-effect profiles of the monoclonal antibodies elotuzumab and daratumumab ${ }^{51}$ and have also revealed synergy between these agents and the IMiDs. ${ }^{52}$ Thus, there is significant interest in incorporating these agents into postASCT maintenance strategies as they are predicted to improve long-term outcomes without contributing significant toxicity. Kinesin spindle inhibition (NCT01372540), selective histone deacetylase inhibition (NCT01323751), or nuclear transport inhibition (NCT02336815) are some of the newer agents in clinical trials which warrant future investigation. Newer treatment strategies such as checkpoint inhibition may also prove beneficial in the post-ASCT setting. Recent studies have demonstrated activity when agents such as pembrolizumab (anti-PD1 antibody) have been combined with IMiDs in the relapsed/refractory setting. ${ }^{53}$ Finally, it is tempting to speculate that the monoclonal antibody NEOD001, which targets misfolded proteins and induces organ responses in $\mathrm{AL}$ amyloidosis, ${ }^{43}$ could be incorporated into induction regimens as well as in the maintenance setting following ASCT to enhance long-term outcomes in AL.

\section{Summary and recommendations}

The use of ASCT as consolidation following induction therapy for myeloma remains a standard of care. Whether there are subgroups of patients who benefit from a tandem ASCT strategy in today's era of novel agents or who do not require ASCT remains to be determined. For patients who relapse at least 18 months following their first ASCT, and who remain transplant-eligible, salvage ASCT should be considered. Three randomized phase 3 studies have demonstrated that lenalidomide maintenance post-ASCT improves outcomes and, in particular, CALGB 100104 has established that lenalidomide maintenance continued until disease progression is a standard of care. The incorporation of newer agents such as oral PIs and monoclonal antibodies into post-ASCT treatment strategies holds the promise of significantly improving outcomes. Ongoing and planned clinical trials will help determine whether a more individualized approach to postASCT therapy can be developed.

\section{Correspondence}

Philip L. McCarthy, Department of Medicine, Roswell Park Cancer Institute, Elm and Carlton Sts, Buffalo, NY 14263; e-mail: philip. mccarthy@ roswellpark.org.

\section{References}

1. Rajkumar SV, Dimopoulos MA, Palumbo A, et al. International Myeloma Working Group updated criteria for the diagnosis of multiple myeloma. Lancet Oncol. 2014;15(12):e538-e548.

2. Wildes TM, Finney JD, Fiala M, et al. High-dose therapy and autologous stem cell transplant in older adults with multiple myeloma. Bone Marrow Transplant. 2015;50(8):1075-1082.
3. Attal M, Harousseau JL, Stoppa AM, et al. A prospective, randomized trial of autologous bone marrow transplantation and chemotherapy in multiple myeloma. Intergroupe Français du Myélome. N Engl J Med. 1996;335(2):91-97.

4. Child JA, Morgan GJ, Davies FE, et al; Medical Research Council Adult Leukaemia Working Party. High-dose chemotherapy with hematopoietic stemcell rescue for multiple myeloma. $N$ Engl J Med. 2003;348(19):1875-1883.

5. Palumbo A, Cavallo F, Gay F, et al. Autologous transplantation and maintenance therapy in multiple myeloma. $N$ Engl J Med. 2014;371(10):895-905.

6. Gay F, Oliva S, Petrucci MT, et al. Chemotherapy plus lenalidomide versus autologous transplantation, followed by lenalidomide plus prednisone versus lenalidomide maintenance, in patients with multiple myeloma: a randomised, multicentre, phase 3 trial. Lancet Oncol. 2015;16(16): 1617-1629.

7. Attal M, Lauwers-Cances V, Hulin C, et al. Autologous transplantation for multiple myeloma in the era of new drugs: a phase III study of the Intergroupe Francophone Du Myelome (IFM/DFCI 2009 trial) [abstract]. Blood. 2015;126(23). Abstract 391.

8. Cavo M, Palumbo A, Zweegman S, et al. Upfront autologous stem cell transplantation (ASCT) versus novel agent-based therapy for multiple myeloma (MM): a randomized phase 3 study of the European Myeloma Network (EMN02/HO95 MM trial) [abstract]. J Clin Oncol. 2016; 34(suppl). Abstract 8000.

9. Gay F, Magarotto V, Spencer A, et al. Upfront or rescue transplant in young patients with newly diagnosed multiple myeloma: a pooled analysis of 529 patients. Haematologica. 2016;101:s104.

10. Barlogie B, Jagannath S, Desikan KR, et al. Total therapy with tandem transplants for newly diagnosed multiple myeloma. Blood. 1999;93(1):55-65.

11. Kumar A, Kharfan-Dabaja MA, Glasmacher A, Djulbegovic B. Tandem versus single autologous hematopoietic cell transplantation for the treatment of multiple myeloma: a systematic review and meta-analysis. J Natl Cancer Inst. 2009;101(2):100-106.

12. Naumann-Winter F, Greb A, Borchmann P, Bohlius J, Engert A, Schnell R. First-line tandem high-dose chemotherapy and autologous stem cell transplantation versus single high-dose chemotherapy and autologous stem cell transplantation in multiple myeloma, a systematic review of controlled studies. Cochrane Database Syst Rev. 2012;10:CD004626.

13. Mai EK, Benner A, Bertsch U, et al. Single versus tandem high-dose melphalan followed by autologous blood stem cell transplantation in multiple myeloma: long-term results from the phase III GMMG-HD2 trial. Br J Haematol. 2016;173(5):731-741.

14. Holstein SA, Richardson PG, Laubach JP, McCarthy PL. Management of relapsed multiple myeloma after autologous stem cell transplant. Biol Blood Marrow Transplant. 2015;21(5):793-798.

15. Michaelis LC, Saad A, Zhong X, et al; Plasma Cell Disorders Working Committee of the Center for International Blood and Marrow Transplant Research. Salvage second hematopoietic cell transplantation in myeloma. Biol Blood Marrow Transplant. 2013;19(5):760-766.

16. Giralt S, Garderet L, Durie B, et al; American Society of Blood and Marrow Transplantation; European Society of Blood and Marrow Transplantation; Blood and Marrow Transplant Clinical Trials Network; International Myeloma Working Group Consensus Conference. American Society of Blood and Marrow Transplantation, European Society of Blood and Marrow Transplantation, Blood and Marrow Transplant Clinical Trials Network, and International Myeloma Working Group Consensus Conference on Salvage Hematopoietic Cell Transplantation in Patients with Relapsed Multiple Myeloma. Biol Blood Marrow Transplant. 2015;21(12):2039-2051.

17. Cook G, Ashcroft AJ, Cairns DA, et al; National Cancer Research Institute Haemato-oncology Clinical Studies Group. The effect of salvage autologous stem-cell transplantation on overall survival in patients with relapsed multiple myeloma (final results from BSBMT/UKMF Myeloma $\mathrm{X}$ Relapse [Intensive]): a randomised, open-label, phase 3 trial. Lancet Haematol. 2016;3(7):e340-351.

18. Dhakal B, Vesole DH, Hari PN. Allogeneic stem cell transplantation for multiple myeloma: is there a future? Bone Marrow Transplant. 2016; 51(4):492-500.

19. Krishnan A, Pasquini MC, Logan B, et al; Blood Marrow Transplant Clinical Trials Network (BMT CTN). Autologous haemopoietic stemcell transplantation followed by allogeneic or autologous haemopoietic 
stem-cell transplantation in patients with multiple myeloma (BMT CTN 0102): a phase 3 biological assignment trial. Lancet Oncol. 2011;12(13): 1195-1203.

20. Garban F, Attal M, Michallet M, et al. Prospective comparison of autologous stem cell transplantation followed by dose-reduced allograft (IFM99-03 trial) with tandem autologous stem cell transplantation (IFM99-04 trial) in high-risk de novo multiple myeloma. Blood. 2006; 107(9):3474-3480.

21. Bruno B, Rotta M, Patriarca F, et al. A comparison of allografting with autografting for newly diagnosed myeloma. N Engl J Med. 2007;356(11): 1110-1120.

22. Gahrton G, Iacobelli S, Björkstrand B, et al; EBMT Chronic Malignancies Working Party Plasma Cell Disorders Subcommittee. Autologous/reducedintensity allogeneic stem cell transplantation vs autologous transplantation in multiple myeloma: long-term results of the EBMT-NMAM2000 study. Blood. 2013;121(25):5055-5063.

23. Cavo M, Tacchetti P, Patriarca F, et al; GIMEMA Italian Myeloma Network. Bortezomib with thalidomide plus dexamethasone compared with thalidomide plus dexamethasone as induction therapy before, and consolidation therapy after, double autologous stem-cell transplantation in newly diagnosed multiple myeloma: a randomised phase 3 study. Lancet. 2010;376(9758):2075-2085.

24. Mellqvist UH, Gimsing P, Hjertner O, et al; Nordic Myeloma Study Group. Bortezomib consolidation after autologous stem cell transplantation in multiple myeloma: a Nordic Myeloma Study Group randomized phase 3 trial. Blood. 2013;121(23):4647-4654.

25. Straka C, Vogel M, Muller J, et al. Results from two phase III studies of bortezomib (BTZ) consolidation vs observation (OBS) post-transplant in patients (pts) with newly diagnosed multiple myeloma (NDMM) [abstract]. J Clin Oncol. 2015;33(suppl). Abstract 8511.

26. Fritz E, Ludwig H. Interferon-alpha treatment in multiple myeloma: meta-analysis of 30 randomised trials among 3948 patients. Ann Oncol. 2000;11(11):1427-1436.

27. Myeloma Trialists' Collaborative Group. Interferon as therapy for multiple myeloma: an individual patient data overview of 24 randomized trials and 4012 patients. Br J Haematol. 2001;113(4):1020-1034.

28. McCarthy PL, Palumbo A. Maintenance therapy for multiple myeloma. Hematol Oncol Clin North Am. 2014;28(5):839-859.

29. Morgan GJ, Davies FE, Gregory WM, et al. Long-term follow-up of MRC Myeloma IX trial: Survival outcomes with bisphosphonate and thalidomide treatment. Clin Cancer Res. 2013;19(21):6030-6038.

30. Attal M, Palumbo A, Holstein SA, et al. Lenalidomide (LEN) maintenance (MNTC) after high-dose melphalan and autologous stem cell transplant (ASCT) in multiple myeloma (MM): a meta-analysis (MA) of overall survival (OS) [abstract]. J Clin Oncol. 2016;34(suppl). Abstract 8001.

31. McCarthy PL, Owzar K, Hofmeister CC, et al. Lenalidomide after stemcell transplantation for multiple myeloma. N Engl J Med. 2012;366(19): 1770-1781.

32. Holstein SA, Owzar K, Richardson PG, et al. Updated analysis of CALGB/ ECOG/BMT CTN 100104: lenalidomide (Len) vs. placebo (PBO) maintenance therapy after single autologous stem cell transplant (ASCT) for multiple myeloma (MM) [abstract]. J Clin Oncol. 2015;33(suppl). Abstract 8523.

33. Attal M, Lauwers-Cances V, Marit G, et al; IFM Investigators. Lenalidomide maintenance after stem-cell transplantation for multiple myeloma. N Engl J Med. 2012;366(19):1782-1791.

34. Attal M, Lauwers-Cances V, Marit G, et al. Lenalidomide maintenance after stem-cell transplantation for multiple myeloma: follow-up analysis of the IFM 2005-02 trial [abstract]. Blood. 2013;122(21). Abstract 406.

35. Rajkumar SV, Jacobus S, Callander NS, et al; Eastern Cooperative Oncology Group. Lenalidomide plus high-dose dexamethasone versus lenalidomide plus low-dose dexamethasone as initial therapy for newly diagnosed multiple myeloma: an open-label randomised controlled trial. Lancet Oncol. 2010;11(1):29-37.

36. Mullighan CG, Su X, Zhang J, et al; Children's Oncology Group. Deletion of IKZF1 and prognosis in acute lymphoblastic leukemia. $N$ Engl $J$ Med. 2009;360(5):470-480.

37. Sonneveld P, Schmidt-Wolf IG, van der Holt B, et al. Bortezomib induction and maintenance treatment in patients with newly diagnosed multiple myeloma: results of the randomized phase III HOVON-65/ GMMG-HD4 trial. J Clin Oncol. 2012;30(24):2946-2955.

38. Sonneveld P, Salwender H-J, Van Der Holt B, et al. Bortezomib induction and maintenance in patients with newly diagnosed multiple myeloma: long-term follow-up of the HOVON-65/GMMG-HD4 Trial [abstract]. Blood. 2015;126(23). Abstract 27.

39. Dispenzieri A. Myeloma: management of the newly diagnosed high-risk patient. Hematology Am Soc Hematol Educ Program. 2016;2016:485-494.

40. Sher T, Dispenzieri A, Gertz MA. Evolution of hematopoietic cell transplantation for immunoglobulin light chain amyloidosis. Biol Blood Marrow Transplant. 2016;22(5):796-801.

41. Palladini G, Merlini G. What is new in diagnosis and management of light chain amyloidosis? Blood. 2016;128(2):159-168.

42. Jaccard A, Moreau P, Leblond V, et al; Myélome Autogreffe (MAG) and Intergroupe Francophone du Myélome (IFM) Intergroup. High-dose melphalan versus melphalan plus dexamethasone for AL amyloidosis. N Engl J Med. 2007;357(11):1083-1093.

43. Gertz MA, Landau H, Comenzo RL, et al. First-in-human phase I/II study of NEOD001 in patients with light chain amyloidosis and persistent organ dysfunction. J Clin Oncol. 2016;34(10):1097-1103.

44. Sanchorawala V, Hoering A, Seldin DC, et al. Modified high-dose melphalan and autologous SCT for AL amyloidosis or high-risk myeloma: analysis of SWOG trial S0115. Bone Marrow Transplant. 2013;48(12):1537-1542.

45. Wechalekar A, Whelan C, Lachmann H, et al. Oral doxycycline improves outcomes of stage III AL amyloidosis-a matched case control study [abstract]. Blood. 2015;126(23). Abstract 732.

46. Kumar SK, Dispenzieri A, Lacy MQ, et al. Doxycycline used as post transplant antibacterial prophylaxis improves survival in patients with light chain amyloidosis undergoing autologous stem cell transplantation [abstract]. Blood. 2012;120(21). Abstract 3138.

47. Reece DE, Hegenbart U, Sanchorawala V, et al. Efficacy and safety of onceweekly and twice-weekly bortezomib in patients with relapsed systemic AL amyloidosis: results of a phase 1/2 study. Blood. 2011;118(4):865-873.

48. Mikhael JR, Schuster SR, Jimenez-Zepeda VH, et al. Cyclophosphamidebortezomib-dexamethasone (CyBorD) produces rapid and complete hematologic response in patients with AL amyloidosis. Blood. 2012;119(19): 4391-4394.

49. Sanchorawala V, Quillen K, Sloan JM, Andrea NT, Seldin DC. Bortezomib and high-dose melphalan conditioning for stem cell transplantation for $\mathrm{AL}$ amyloidosis: a pilot study. Haematologica. 2011;96(12):1890-1892.

50. Kumar S, Berdeja JG, Niesvizky R, et al. Long-term ixazomib maintenance is tolerable and improves depth of response following ixazomiblenalidomide-dexamethasone induction in patients (Pts) with previously untreated multiple myeloma (MM): phase 2 study results [abstract]. Blood. 2014;124(21). Abstract 82.

51. van de Donk NW, Moreau P, Plesner T, et al. Clinical efficacy and management of monoclonal antibodies targeting CD38 and SLAMF7 in multiple myeloma. Blood. 2016;127(6):681-695.

52. Plesner T, Arkenau H-T, Gimsing P, et al. Daratumumab in combination with lenalidomide and dexamethasone in patients with relapsed or relapsed and refractory multiple myeloma: updated results of a phase $1 / 2$ study (GEN503) [abstract]. Blood. 2015;126(23). Abstract 507.

53. San Miguel J, Mateos M-V, Shah JJ, et al. Pembrolizumab in combination with lenalidomide and low-dose dexamethasone for relapsed/ refractory multiple myeloma (RRMM): Keynote-023 [abstract]. Blood. 2015;126(23). Abstract 505.

54. Attal M, Harousseau JL, Facon T, et al; InterGroupe Francophone du Myélome. Single versus double autologous stem-cell transplantation for multiple myeloma. N Engl J Med. 2003;349(26):2495-2502.

55. Cavo M, Tosi P, Zamagni E, et al. Prospective, randomized study of single compared with double autologous stem-cell transplantation for multiple myeloma: Bologna 96 clinical study. J Clin Oncol. 2007;25(17):2434-2441.

56. Cavo M, Pantani L, Petrucci MT, et al; GIMEMA (Gruppo Italiano Malattie Ematologiche dell'Adulto) Italian Myeloma Network. Bortezomibthalidomide-dexamethasone is superior to thalidomide-dexamethasone as consolidation therapy after autologous hematopoietic stem cell transplantation in patients with newly diagnosed multiple myeloma. Blood. 2012;120(1):9-19. 